\title{
Adult Undifferentiated High Grade Pleomorphic Sarcoma of Bone
}

National Cancer Institute

\section{Source}

National Cancer Institute. Adult Undifferentiated High Grade Pleomorphic Sarcoma of

Bone. NCl Thesaurus. Code C114782.

An undifferentiated high grade pleomorphic sarcoma that arises from the bone and occurs during adulthood. 